\title{
Energy-efficient off-body communication nodes with receive diversity
}

\author{
Patrick Van Torre ${ }^{* \dagger}$, Peter Vanveerdeghem ${ }^{* \ddagger}$, Hendrik Rogier* and Ingrid Moerman ${ }^{\S}$ \\ * INTEC - IMEC, Ghent University \\ Sint-Pietersnieuwstraat 41, 9000 Ghent, Belgium \\ Email: patrick.vantorre@ugent.be \\ Tel: +32 $92643316 \quad$ Fax: +32 92643593 \\ $\S$ iMinds, Ghent University \\ Gaston Crommenlaan 8/201, 9050 Ghent, Belgium \\ $\dagger$ IC\&T GEN, Ghent University \\ Valentin Vaerwyckweg 1, 9000 Ghent, Belgium \\ $\ddagger$ ISP ELIT, Ghent University \\ Graaf Karel de Goedelaan 5, 8500 Kortrijk, Belgium
}

\begin{abstract}
Off-body wireless communication applications range from fall-detection systems for the elderly to monitoring networks for rescue workers. Further development of practical body-worn systems requires compact, low-cost and low-power battery-powered equipment. A versatile wearable network node offering all these features, including a powerful microcontroller for data processing and additional memory for local data logging was designed and implemented. The node allows receive diversity, mitigating the negative impact of fading, which is typically present in indoor propagation environments. Channel measurements are performed for an indoor Non Line-of-Sight communication between two nodes. Mobile-to-base-station as well as mobile-to-mobile links are considered. A statistical analysis of the performance determines outage probability with and without receiver diversity for both link types, showing a significant diversity gain in all cases. Correlation properties, level crossing rate and average fade duration are also determined.
\end{abstract}

\section{INTRODUCTION}

Off-body communication is an important research topic, with many applications such as fall-detection for the elderly and rescue worker monitoring. Further potential applications include military and law enforcement operations as well as baby monitoring. Off-body communication systems including sensors transmit real-time vital information towards a fixed base-station or other mobile off-body network nodes. A multitude of sensors are interesting for body-centric applications, such as accelerometers, gyroscopes, magnetometers as well as sensors measuring temperature, humidity and heart rate [1]. All these sensors collect information about the condition of the user.

These systems are mostly employed in indoor environments, where the radio propagation is influenced by fading and shadowing. Propagation conditions can be Line-of-Sight (LoS) or Non Line-of-Sight (NLoS), the latter one referring to a signal path that is obstructed by walls or objects causing severe attenuation. The performance of a basic wireless communication system is highly compromised under NLoS conditions. It is well known that in NLoS, fading alone contributes to received signal variations up to $35 \mathrm{~dB}$. For off-body communication, fading occurs together with shadowing by the environment as well as by the user's body, causing additional signal variation. Despite the effects described above, the human body is an excellent platform for a wireless communication system, as the large surface area permits to deploy multiple antennas. The detrimental impact of fading and shadowing on the link quality is effectively countered by employing diversity techniques to combine the signals of several on-body antennas.

In literature a large number of measurement campaigns for off-body communication with diversity techniques are documented, in most cases employing bulky equipment connected to on-body antennas using long coaxial cables. We designed and implemented a compact low-cost energy-efficient network node that is directly connected to on-body textile antennas, similar to those previously used in [6], by means of short coaxial cables. Only one feed of these dual-polarized antennas is used, resulting in a $45^{\circ}$ slanted linear polarization for each antenna. The system, including a suitable battery, can be easily integrated into a garment and is hence fully wearable in a nonobtrusive way.

The novel wearable node contains a number of integrated circuits as key components, including a state-of-theart $2.45 \mathrm{GHz}$ wireless transceiver and microcontroller, as well as a 3-axis accelerometer and $128 \mathrm{~KB}$ flash memory for onboard storage of measurement results. Compared to the offbody $2.45 \mathrm{GHz}$ units described earlier in [2]-[5], the new circuit design described here employs the novel ADF7242 integrated transceiver by Analog Devices, directly allowing diversity reception for the first time and never described for design of off-body communication systems in literature before.

Second-order receiver diversity is implemented by means of Selection Combining. A fully digital interface to a powerful microcontroller enables local processing and storage of information. The microcontroller allows to implement a network protocol stack in order to make the wearable network nodes forward packets towards each other, setting up an ad hoc wireless network where each node functions as a repeater. 
The paper is organized as follows. Section II describes the system in more detail, whereas in Section III a number of measurement results for the system is presented. Section IV provides more insight into the behavior of the system for communication between mobile nodes as well as for a link to a base station. The conclusions are presented in Section V.

\section{DESCRIPTION OF THE SYSTEM}

As illustrated in the block diagram in Fig. 2, the main component of the wireless node is the ADF7242 transceiver from Analog Devices. This is a very recent component allowing communication in the $2.45 \mathrm{GHz}$ license-free I.S.M. (Industrial, Scientific and Medical) band using IEEE 802.15.4 compliant transmissions as well as GFSK modulation with a data rate up to $2 \mathrm{Mbps}$. The transceiver chip is low-power and very flexible to use. The IEEE 802.15.4 mode in particular allows reliable communication including forward error correction. The transceiver includes two RF ports, allowing a single unit to be connected to front and back textile antennas worn on the body to form a spatial- and pattern-diversity system.

The textile antenna used is displayed in Fig. 1 and is made completely out of flexible materials. For the radiating patch, ShieldIt ${ }^{\circledR}$ is used, whereas for the ground plane FlecTron ${ }^{\circledR}$ is employed, two highly conductive electrotextile materials. The isolating substrate is a breathable moisture- and fire-resistant foam, also used as a thermal isolating layer in firefighter garments. The patch antenna consists of a rectangular patch with a slot. Two feed points are provided, for orthogonal linear polarizations of $\pm 45^{\circ}$. In the measurement presented here, only on polarization is used by connecting one port to the transceiver and the other port to a $50 \Omega$ load. The radiation patterns of the antenna are documented in [6].

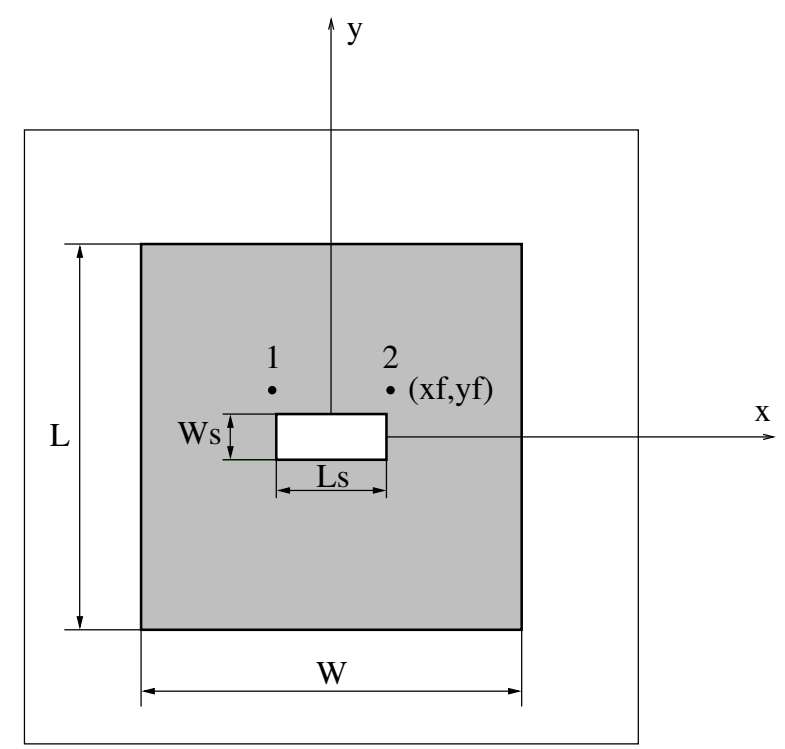

air

patch

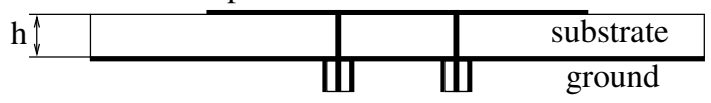

Fig. 1. Block diagram of the wireless sensor node
Other key components include a C8051F921 microcontroller by Silicon Laboratories. This controller is in-circuit programmable (ISP) using a proprietary 2 -wire interface, provides 25 MIPS peak throughput and can operate on a supply voltage as low as 0.9 Volt. A $32 \mathrm{~KB}$ flash memory chip is provided to store programs, as well as $4 \mathrm{~KB}$ of internal RAM. To provide local nonvolatile storage space for measurement purposes, an extra $128 \mathrm{~KB}$ of flash memory is included on the board.

By way of example, a 3-axis accelerometer was included in the prototypes. Yet, the microcontroller has many analog and digital inputs, allowing the connection of virtually any type of sensor. The accelerometer is very compact and provides interesting information about the user's movements and posture.

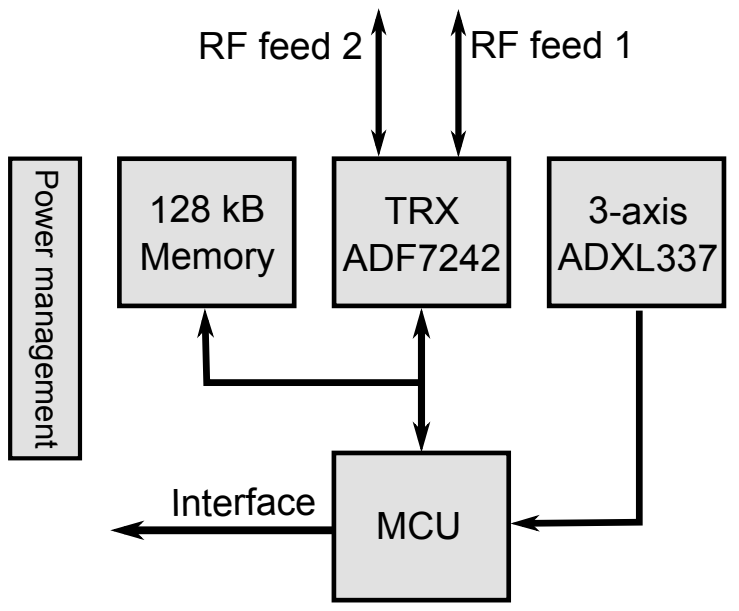

Fig. 2. Block diagram of the wireless sensor node

The circuit is implemented on a standard FR4 printed circuit board, as illustrated in Fig. 3. A pair of SMA-connectors is installed near the right-side edge of the board, allowing connection of antennas by means of coaxial cables.

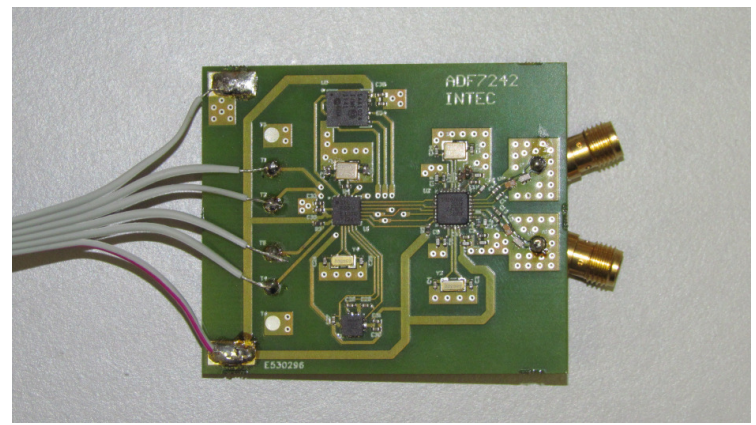

Fig. 3. Picture of the wireless sensor node. The flatcable is only used during software development.

\section{MeAsurement CAMPAign}

\section{A. Measurement setup}

Measurements are performed in an indoor office environment at Ghent University. The building consists of solid brick walls and reinforced concrete floors, causing severe attenuation of signals in the $2.45 \mathrm{GHz}$ band and true NLoS propagation, as observed in multiple previous measurement campaigns, most recently documented in [7]. 
Communication is set up between offices at a distance of $15 \mathrm{~m}$, as illustrated in Fig. 4, yielding a NLoS link with negligible packet loss for accurate signal level measurements. Note that the system's range is much larger, especially for low data rates.

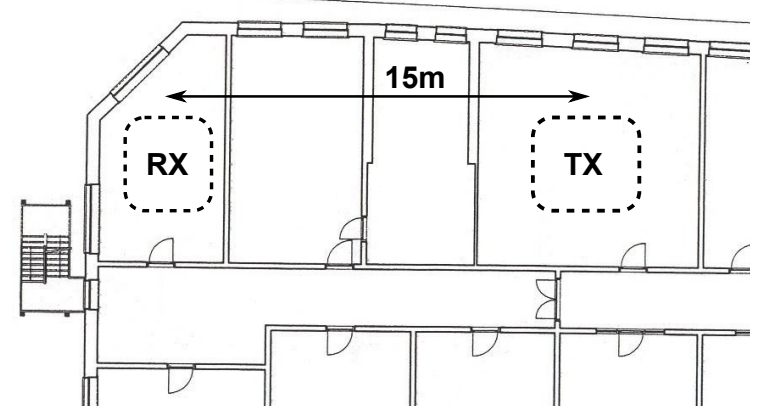

Fig. 4. Floor plan of the indoor environment

Two measurement series are performed, for a fixed and mobile transmitter.

- For the fixed location the textile patch antenna is taped to a cardboard box on a table in the office, resulting in a static antenna at equal height above the floor as if worn on the human body.

- For the mobile locations, the antennas are taped on the center of the front and back of the test person's torso. In receive mode both antennas are used, whereas in transmit mode only the front antenna is active.

The ADF7242 transmitter is configured in IEEE802.15.4 mode at its maximum power of $4.8 \mathrm{dBm}$ and transmits 100 packets per second. Each packet contains a unique serial number, in order to enable the assessment of potential packet loss at the receiver.

The mobile receiver using front and back antennas connected to a single unit, switches between these antennas after each received frame. Odd frames are received on the front antenna and even frames at the back antenna. Hence, for each antenna, a signal level is recorded each $20 \mathrm{~ms}$, which is a period shorter than the $2.45 \mathrm{GHz}$ indoor channel's coherence time for a walking person, determined to be at least $21.2 \mathrm{~ms}$ in [8] and [9]. The autocorrelation values discussed in Section IV will provide further clarification.

Signal levels for a walking person in an indoor environment are influenced by many factors, including fading and shadowing by the environment, changing path loss, shadowing by the human body as well as reorientation of antenna radiation patterns. The influence of path loss and shadowing is minimized by limiting the area covered by the test persons to a few square meters. This displacement is more than enough to cause severe fading at $2.45 \mathrm{GHz}$, where the wavelength is only $12 \mathrm{~cm}$. Note that each walking person also randomly reorients during the complete measurement.

\section{B. Signal levels for a mobile to base-station link}

Received signal levels for the first 10 seconds of the mobile to base-station measurement are illustrated in Fig. 5. Note that the total measurement stores $163.84 \mathrm{~s}$ of communication, resulting in 8192 recorded packets per receiving antenna. A limited timespan was chosen for graph clarity reasons only.

Signal levels rapidly fluctuate over a $30 \mathrm{~dB}$ range, partially independent on front and back antennas. Hence, a significant diversity gain is possible by combining the signals, as further illustrated.

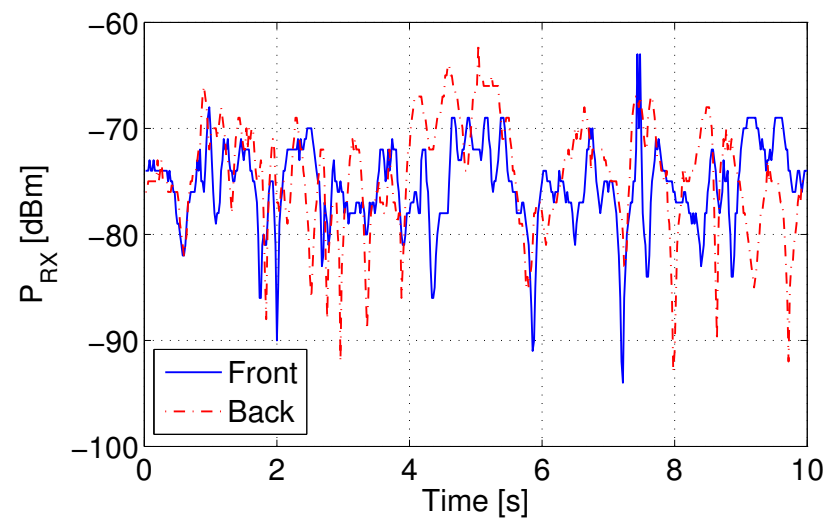

Fig. 5. Behavior of the received signals on front and back antennas for a NLoS link between a walking person and a fixed base station

Fig. 6 displays similar results for the mobile-to-mobile link. The average received power is $3.0315 \mathrm{~dB}$ lower than for the mobile-to-fixed link, due to the directional radiation pattern of the on-body transmitting antenna, covering approximately a half-space around the body. During the random orientation of the walking person, the transmitting antenna is expected to be oriented away from the receiver approximately half of the time, whereas for a fixed station the antenna was always oriented towards the receiver (although in NLoS conditions).

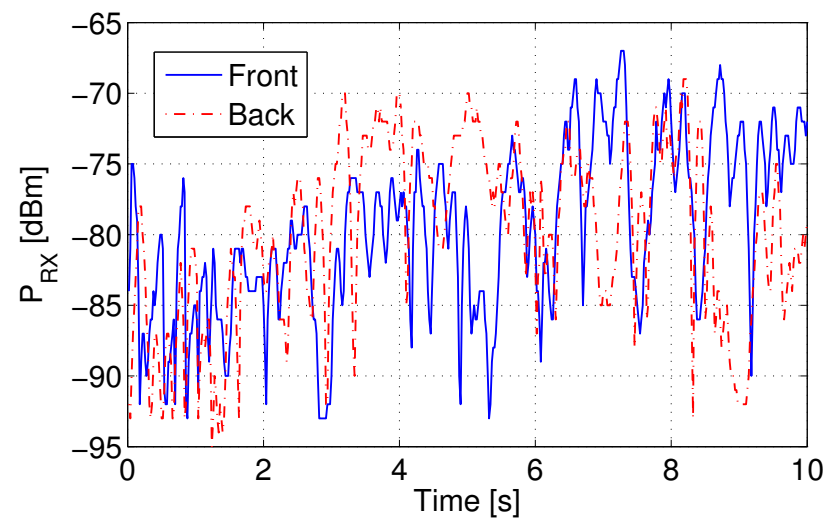

Fig. 6. Behavior of the received signals on front and back antennas for a NLoS link between two walking persons

\section{Statistical ANALYsis}

Statistical analysis of the 8192 recorded samples per receiving antenna allows accurate extraction of the Cumulative Distribution Functions (CDF) in order to determine the $10 \%$ outage probability levels with and without diversity reception. 


\section{A. Mobile-to-Fixed Link CDF}

The CDF for the mobile-to-fixed link is displayed on a logarithmic scale in Fig. 7. CDF curves for the front and back antennas are very similar and match the theoretical Rayleigh fading CDF calculated as $1-\exp \left(-r^{2} / 2 \sigma^{2}\right)$, with $r$ the signal envelope and $\sigma$ determined by setting $\sigma \sqrt{(} \pi / 2)$ equal to the mean signal envelope for the full measurement on both antennas. Results with diversity are compared to simulated characteristics for equal gain uncorrelated Rayleigh fading channels. The downward shift of the measured curves for lower received powers is attributed to the limited number of samples.

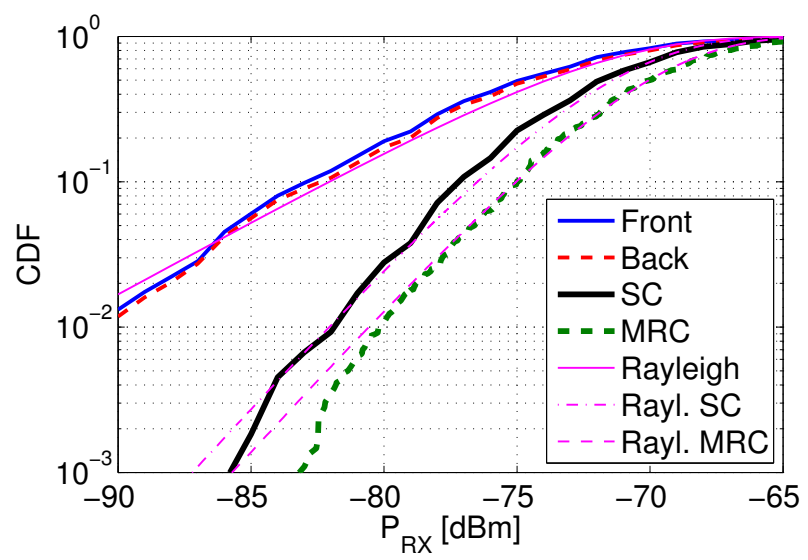

Fig. 7. CDF for the link to the fixed base station for the signals on front and back antennas; combined signals with SC and MRC ; theoretical Rayleigh characteristic

Selection Combining (SC) provides receiver diversity gain. This technique can be implemented directly by the microcontroller. The transmitter has to send the same payload twice, in two subsequent packets, to allow practical data reception with SC. The microcontroller will then select from each received pair of packets the one with the highest received power. The $\mathrm{CDF}$ for SC shows a $10 \%$ outage probability improvement of $5 \mathrm{~dB}$. Hence, $90 \%$ of the time the signal level will be above $-77.5 \mathrm{dBm}$ for $\mathrm{SC}$, compared to $-82.5 \mathrm{dBm}$ for a single input. Future integrated transceivers employing Maximum Ratio Combining (MRC) will provide a 10\% outage probability improvement of $7.5 \mathrm{~dB}$ in the same situation. This result is calculated by adding the received powers on both inputs, assuming equal noise floors for both inputs.

\section{B. Mobile-to-Mobile Link CDF}

The CDF curves for the Mobile-to-Mobile link are shown in Fig. 8. The characteristics are again very similar for front and back antennas. However, due to the Double Rayleigh fading occurring at both link ends [10], [11], the curves are significantly different from the theoretical Rayleigh characteristic. However, because of the more serious fading, the $10 \%$ outage probability level now improves by $7.5 \mathrm{~dB}$ for SC en by $8.5 \mathrm{~dB}$ for MRC, illustrating the benefit of front-to-back receiver diversity for both scenario's.

According to [11], the CDF for the Double Rayleigh process is defined as

$$
F(r)=1-\frac{r}{\sigma_{1} \sigma_{2}} K_{1}\left(\frac{r}{\sigma_{1} \sigma_{2}}\right), r \geq 0
$$

with $K_{1}(\cdot)$ the first order modified Bessel function of the second kind, $r$ the signal envelope and $\sigma_{1} \sigma_{2}=8.9 \cdot 10^{-5}$. The Double Rayleigh fading CDF's match the measurement for the higher signal levels, where the curves are not influenced by packet loss.

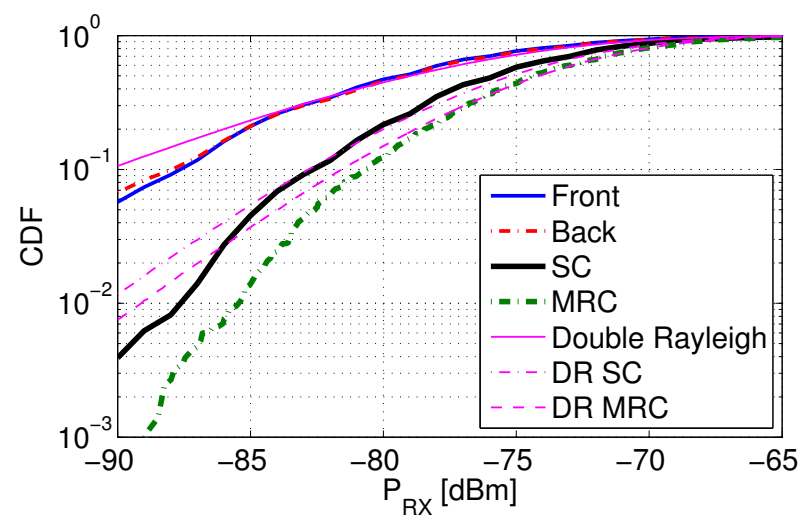

Fig. 8. CDF for the link between two mobile users for the signals on front and back antennas; combined signals with SC and MRC; theoretical Double Rayleigh fading characteristic

\section{Correlation Properties}

The envelope correlation coefficients for front and back antennas are equal to 0.0853 for the mobile-to-fixed link and 0.0844 for the mobile-to-mobile link. These very low correlation values illustrate the independence of the signals on front and back receiving antennas in NLoS conditions. The complementarity of two antennas on opposite sides of the body is increased by body shadowing as well as by oppositely oriented directional radiation patterns. Therefore receiver diversity is guaranteed to provide a substantial gain.

Arguably, the signal envelope values for both antennas are captured with $10 \mathrm{~ms}$ time difference, potentially resulting in a small degree of time diversity. However, in Fig. 9, the autocorrelation functions for the two antenna signals in both scenarios illustrate that a highly significant correlation is present up to a much larger time shift. Hence, the influence of the measurement time difference on the spatial and pattern diversity gain is assumed to be negligible.

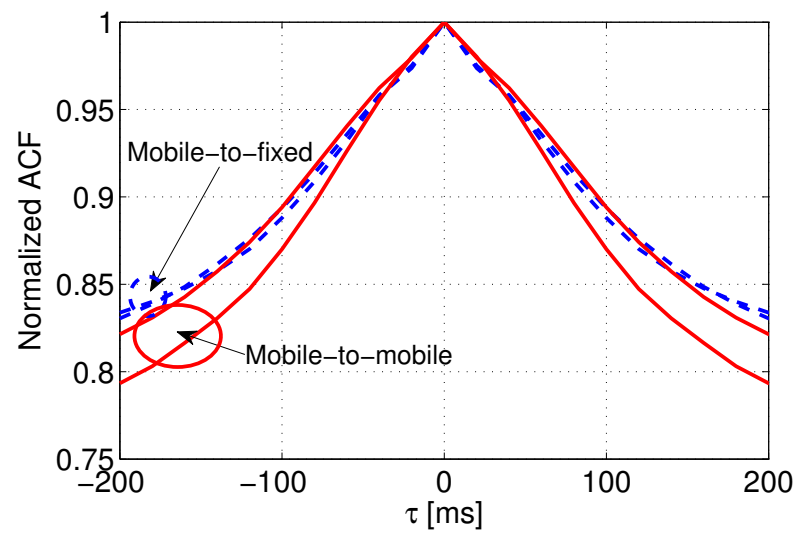

Fig. 9. Autocorrelation function for both antennas in both scenarios 


\section{Level Crossing Rate}

The Level Crossing Rate (LCR) is a 2nd-order statistical parameter describing the number of times the normalized signal envelope crosses a given threshold level $r$ with a positive slope, illustrating the speed of fading. The LCR is determined for the measurements by an algorithm in MATLAB, counting the number of level crossings for the 8192 recorded signal levels for each antenna.

The number of level crossings per second is displayed as a function of relative received power in Fig.10 for both measurement scenarios. The LCR characteristics are very similar for both antennas in the same scenario, whereas for different scenarios the shape of the curves is slightly different.

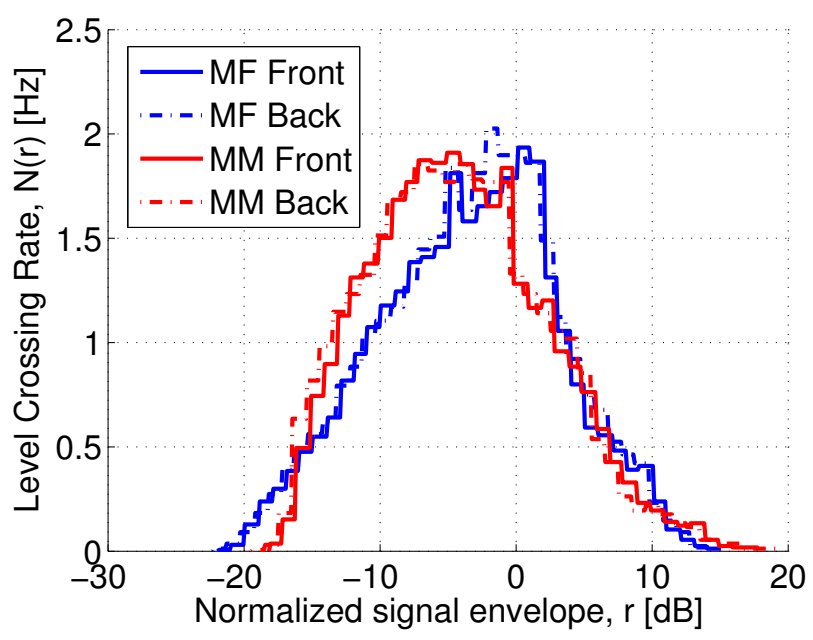

Fig. 10. Level Crossing Rate (LCR) for front and back antennas of the mobile-to-fixed (MF) and mobile-to-mobile (MM) links

Surprisingly, the peak LCR values are not very different for both scenarios, contradictory to [12] which states that a Double Rayleigh-fading channel will fade twice as often as a normal Rayleigh fading channel. However, for the mobile-to-mobile link the value can be influenced by many factors, including the structure of the environment, the reorientation of the directional transmitting antenna and the limited measurement time.

\section{E. Average Duration of Fading}

The Average Duration of Fading (ADF) is related to the LCR and expresses the average period of time the normalized signal envelope is below a given threshold level $r$. The ADF for both measurement scenarios is calculated in MATLAB by counting $L(r)$, the number of samples where the signal level is lower than $r$. With $N(r)$ the LCR; $T_{s}=0.02 \mathrm{~s}$ the sampling time and $T_{m}=163.84 \mathrm{~s}$ the total measurement time, the ADF is expressed as $T(r)=L(r) \cdot T_{s} /\left(N(r) \cdot T_{m}\right)$ and displayed in Fig. 11.

All ADF curves are very similar and are useful when considering data interleaving to enhance the reliability of the communication. For the average received signal envelope $(0 \mathrm{~dB}$ in the graph), the average fade lasts approximately $300 \mathrm{~ms}$.

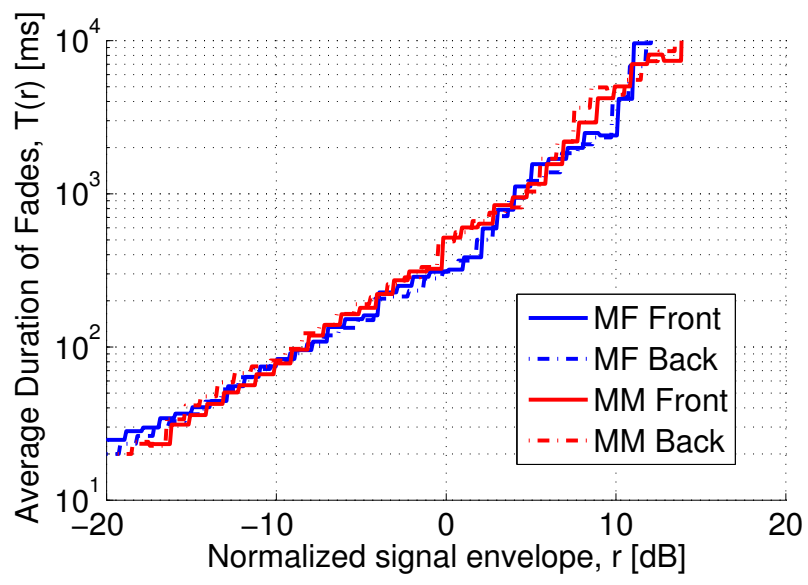

Fig. 11. Average Duration of Fading (ADF) for front and back antennas of the mobile-to-fixed (MF) and mobile-to-mobile (MM) links

\section{CONCLUSION}

The compact off-body low-power wearable wireless communication node provides effective diversity gain by selection combining on the node for mobile-to-base-station as well as for mobile-to-mobile links connecting to walking persons in indoor environments. Statistical analysis illustrates the reliable performance obtained in Non Line-of-Sight scenario's, corresponding to the most difficult propagation conditions. For channel measurement purposes the wireless node provides a high measurement speed, allowing repeated measurements within the channel's coherence time on each antenna. Statistical analysis shows a $5 \mathrm{~dB}$ improvement in $10 \%$ outage probability level for both mobile-to-fixed and mobile-to-mobile links.

\section{ACKNOWLEDGMENT}

This research was funded by the Interuniversity Attraction Poles (IAP phase-VII) program, provided by the Belgian Science Policy Office (BELSPO).

\section{REFERENCES}

[1] M. Patel and Jianfeng Wang, "Applications, challenges, and prospective in emerging body area networking technologies", Wireless Communications, IEEE, vol. 17, no. 1, pp. 80-88, 2010.

[2] S.L. Cotton, G.A. Conway, and W.G. Scanlon, "A Time-Domain Approach to the Analysis and Modeling of On-Body Propagation Characteristics Using Synchronized Measurements at $2.45 \mathrm{GHz}$ ", Antennas and Propagation, IEEE Transactions on, vol. 57, no. 4, pp. 943-955, April 2009.

[3] S. Sharma, A.L. Vyas, B. Thakker, D. Mulvaney, and S. Datta, "Wireless body area network for health monitoring", in Biomedical Engineering and Informatics (BMEI), 2011 4th International Conference on, 2011, vol. 4, pp. 2183-2186.

[4] S.S. Sonavane, V. Kumar, and B.P. Patil, "Designing wireless sensor network with low cost and low power", in Networks, 2008. ICON 2008. 16th IEEE International Conference on, 2008, pp. 1-5.

[5] M.O. Munoz, R. Foster, and Yang Hao, "On-body channel measurement using wireless sensors", Antennas and Propagation, IEEE Transactions on, vol. 60, no. 7, pp. 3397-3406, 2012.

[6] L. Vallozzi, P. Van Torre, C. Hertleer, H. Rogier, M. Moeneclaey, and J. Verhaevert, "Wireless communication for firefighters using dualpolarized textile antennas integrated in their garment", Antennas and Propagation, IEEE Transactions on, vol. 58, no. 4, pp. $1357-1368$, April 2010. 
[7] Patrick Van Torre, Luigi Vallozzi, Lennert Jacobs, Hendrik Rogier, Marc Moeneclaey, and Jo Verhaevert, "Characterization of measured indoor off-body MIMO channels with correlated fading, correlated shadowing and constant path loss", Wireless Communications, IEEE Transactions on, vol. 11, no. 2, pp. 712-721, 2012.

[8] Heather MacLeod, Chris Loadman, and Zhizhang Chen, "Experimental studies of the 2.4-GHz ISM wireless indoor channel", in Communication Networks and Services Research Conference, 2005. Proceedings of the 3rd Annual. IEEE, 2005, pp. 63-68.

[9] Patrick Van Torre, Luigi Vallozzi, Hendrik Rogier, Marc Moeneclaey, and Jo Verhaevert, "Channel characterization and robust tracking for diversity reception over time-variant off-body wireless communication channels", EURASIP Journal on Advances in Signal Processing, p. 14, 2010
[10] Jari Salo, Hassan M El-Sallabi, and Pertti Vainikainen, "Impact of double-rayleigh fading on system performance", in Wireless Pervasive Computing, 2006 1st International Symposium on. IEEE, 2006, pp. 5pp.

[11] Batool Talha and Matthias Pätzold, "On the statistical properties of double rice channels", in Proc. 10th International Symposium on Wireless Personal Multimedia Communications, WPMC 2007, 2007, pp. 517-522.

[12] D. Gesbert, H. Bolcskei, D. Gore, and A. Paulraj, "MIMO wireless channels: capacity and performance prediction", in Global Telecommunications Conference, 2000. GLOBECOM 'O0. IEEE, 2000, vol. 2, pp. $1083-1088$ vol.2. 